\title{
Incorporation of density-matrix wave functions in Monte Carlo simulations: Application to the frustrated Heisenberg model
}

\author{
M. S. L. du Croo de Jongh, J. M. J. van Leeuwen, and W. van Saarloos \\ Instituut-Lorentz, Leiden University, P.O. Box 9506, 2300 RA Leiden, The Netherlands
}

(Received 8 February 2000; revised manuscript received 5 July 2000)

\begin{abstract}
We combine the density-matrix renormalization-group (DMRG) technique with Green function Monte Carlo (GFMC) simulations using a special representation of the DMRG wave function. As a test case we apply the method to the two-dimensional frustrated Heisenberg antiferromagnet. By supplementing the branching in GFMC simulations with stochastic reconfiguration we get a stable simulation with a small variance also in the region where the fluctuations due to the minus sign problem are maximal. The sensitivity of the results to the choice of the guiding wave function is extensively investigated. In agreement with earlier calculations it is found from the DMRG wave function that for small ratios of the next-nearest-to-nearest neighbor coupling strength the system orders as a Néel-type antiferromagnet and for large ratios as a columnar antiferromagnet. The spin stiffness suggests an intermediate regime without magnetic long-range order. The energy curve indicates that the columnar phase is separated from the intermediate phase by a first-order transition. The combination of the DMRG and GFMC techniques allows us to substantiate this picture by calculating also the spin correlations in the system. We observe a pattern of spin correlations in the intermediate regime which is in between dimerlike and plaquette-type ordering, states that have recently been suggested. It is a state with strong dimerization in one direction and weaker dimerization in the perpendicular direction and thus it lacks the square symmetry of the plaquette state.
\end{abstract}

\section{INTRODUCTION}

The density-matrix renormalization-group (DMRG) technique has proved to be a very efficient method to determine the ground-state properties of low-dimensional systems. ${ }^{1}$ For a quantum chain it produces extremely accurate values for the energy and the correlation functions. In two-dimensional systems the calculational effort increases rapidly with the size of the system. The most favorable geometry is that of a long small strip. In practice the width of the strip is limited to around eight to ten lattice sites. Greens function Monte Carlo (GFMC) simulations are not directly limited by the size of the system but by the efficiency of the importance sampling. When the system has a minus sign problem the statistics is ruined in the long run and accurate estimates are impossible. Many proposals ${ }^{2}$ have been made to alleviate or avoid the minus sign problem with varying success, but all of them introduce uncontrollable errors in the sampling. In the DMRG calculation of the wave function the minus sign problem is not manifestly present. In all proposed cures of the minus sign problem the errors decrease when the guiding wave function approaches the ground state.

The idea of this paper is that DMRG wave functions are much better, also for larger systems, than the educated guesses which usually feature as guiding wave functions. Moreover, the DMRG approach is a general technique to construct a wave function without knowing too much about the nature of the ground state, with the possibility to systematically increase the accuracy. Thus DMRG wave functions would do very well when they could be used as guiding functions in the importance sampling of GFMC simulations. There is a complicating factor which prevents a straightforward implementation of this idea due to the fact that inter- esting systems are so large that it is impossible to use a wave function via a lookup table. The value of the wave function in a configuration has to be calculated by an in-line algorithm. This has limited the guiding wave functions to simple expressions which are fast to evaluate. Consequently such guiding wave functions are not an accurate representation of the true ground-state wave function, in particular if the physics of the ground state is not well understood. In this paper we describe a method to read out the DMRG wave function in an efficient way by using a special representation of the DMRG wave function.

A second problem is that a good guiding wave function alleviates the minus sign problem, but cannot remove it as long as it is not exact. We resolve this dilemma by applying the method of stochastic reconfiguration (SR) which has recently been proposed by Sorella. ${ }^{3}$ The viability of our method is tested for the frustrated Heisenberg model.

The behavior of the two-dimensional Heisenberg antiferromagnet has been intriguing for a long time and still is in the center of research. The source of the complexity of the ground state is the large quantum fluctuations which counteract the tendency of classical ordering. The unfrustrated two-dimensional Heisenberg antiferromagnet orders in a Néel state and by numerical methods the properties of this state can be analyzed accurately. ${ }^{4}$ The situation is worse when the interactions are competing as in a two-dimensional square lattice with antiferromagnetic nearest-neighbor $J_{1}$ and next-nearest-neighbor $J_{2}$ coupling. This spin system with continuous symmetry can order in two dimensions at zero temperature, but it is clear that the magnetic order is frustrated by the opposing tendencies of the two types of interaction. The ratio $J_{2} / J_{1}$ is a convenient parameter for the frustration. For small values the system orders antiferromagnetically in a Néel-type arrangement, which accommodates 
the nearest-neighbor interaction. For large ratios a magnetic order in alternating columns of aligned spins (columnar phase) will prevail; in this regime the roles of the two couplings are reversed: the nearest-neighbor interaction frustrates the order imposed by the next-nearest-neighbor interaction. In between, for ratios of the order of 0.5 , the frustration is maximal and it is not clear which sort of ground state results. This problem has been attacked by various methods but not yet by the DMRG technique and only very recently by GFMC simulations. ${ }^{5}$ This paper addresses the issue by studying the spin correlations.

A simple road to the answer is not possible since frustration implies a sign problem which prevents a straightforward use of the GFMC simulation technique. Moreover, the frustration substantially complicates the structure of the groundstate wave function. Generally frustration encourages the formation of local structures such as dimers and plaquettes which are at odds, but not incompatible, with long-range magnetic order. These correlation patterns are the most interesting part of the intermediate phase and the main goal of this investigation.

Many attempts have been made to clarify the situation. Often simple approximations such as mean-field or spinwave theory give useful information about the qualitative behavior of the phase diagram. A fairly sophistocated meanfield theory using the Schwinger boson representation does not give an intermediate phase. ${ }^{6}$ Given the complexity of the phase diagram and the subtlety of the effects it is not clear whether such approximate methods can give in this case a reliable clue to the qualitative behavior of the system.

Exact calculations have been performed on small systems up to size $6 \times 6$ by Schulz et al. ${ }^{7}$ Although this information is very accurate and unbiased to possible phases, the extrapolation to larger systems is a long way, the more so in view of indications that the anticipated finite-size behavior only applies for larger systems. Another drawback of these small systems is that the ground state is assumed to have the full symmetry of the lattice. Therefore the symmetry breaking, associated with the formation of dimers, ladders, or plaquettes, which is typical for the intermediate state, cannot be observed directly.

More convincing are the systematic series expansion as reported recently by Kotov et al. ${ }^{8,9}$ and by Singh et al., ${ }^{10}$ which bear on an infinite system. They start with independent dimers (plaquettes) and study the series expansion in the coupling between the dimers (plaquettes). By the choice of the state, around which the perturbation expansion is made, the type of spatial symmetry breaking is fixed. These studies favor in the intermediate regime the dimer state over the plaquette state. Their dimer state has dimers organized in ladders in which the chains and the rungs have nearly equal strength. So the system breaks translational invariance only in one direction. The energy differences are, however, small and the series is finite, so further investigation is useful. Our simulations yield correlations in good agreement with theirs, but do not confirm the picture of translational invariant ladders. Instead we find an additional weaker symmetry breaking along the ladders, such that we come closer to the plaquette picture.

Very recently Capriotti and Sorella ${ }^{5}$ have carried out a GFMC simulation for $J_{2}=0.5 J_{1}$ and have studied the sus- ceptibilities for orientational and translational symmetry breaking. They conclude that the ground state is a plaquette state with full symmetry between the horizontal and vertical directions.

From the purely theoretical side the problem has been discussed by Read and Sachdev ${ }^{11}$ on the basis of a large spin expansion. From their analysis a scenario emerges in which the Néel phase disappears upon increasing frustration in a continuous way. Then a gapped spatial-inhomogeneous phase with dimerlike correlations appears. For even higher frustration ratios a first-order transition takes place to the columnar phase. Although this scenario is qualitative, without precise location of the phase transition points, it definitively excludes dimer formation in the magnetically ordered Néel and columnar phases. It is remarkable that two quite different order parameters (the magnetic order and the dimer order) disappear simultaneously and continuously on opposite sides of the phase transition. In this scenario, this is taken as an indication of some kind of duality of the two phases.

Given all these predictions it is of utmost interest to further study the nature of the intermediate state. Due to the smallness of the differences in energy between the various possibilities, the energy will not be an ideal test for the phase diagram. Therefore we have decided to focus on the spin correlations as a function of the ratio $J_{2} / J_{1}$. In this paper we first investigate the two-dimensional frustrated Heisenberg model by constructing the DMRG wave function of the ground state for long strips up to a width of eight sites. The ground-state energy and the spin stiffness which are calculated confirm the overal picture described above, but the results are not accurate enough to allow for a conclusive extrapolation to larger systems. Then we study an open 10 $\times 10$ lattice by means of the GFMC technique using DMRG wave functions as the guiding wave function for the importance sampling. The GFMC simulations are supplemented by stochastic reconfiguration as proposed by Sorella ${ }^{3}$ as an extension of the fixed node technique. ${ }^{12}$ This method avoids the minus sign problem by replacing the walkers regularly by a new set of positive sign with the same statistical properties. The first observation is that GFMC improves the energy of the DMRG technique in a substantial and systematic way as can be tested in the unfrustrated model where sufficient information is available from different sources. Second, the spin correlations become more accurate and less dependent on the technique used for constructing the DMRG wave function. The DMRG technique is focused on the energy of the system and less on the correlations. The GFMC technique probes mostly local correlations of the system as all moves are small and correspond to local changes of the configurations. With these spin correlations we investigate the phase diagram for various values of the frustration ratio $J_{2} / J_{1}$.

After giving the definition of the model we briefly describe the DMRG method and its results for the energy and the stiffness. Then we go into more detail about the way the constructed wave functions can be used as guiding wave functions in the GFMC simulation. This is a delicate problem since the full construction of a DMRG wave function takes several hours on a workstation. Therefore we separate off the construction of the wave function and cast it in a form 
where the configurations can be obtained from each other by matrix operations on a vector. So the length of the computation of the wave function in a configuration scales with the square of the number of states included in the DMRG wave function. The remaining sections concern the GFMC simulations and are focused on the correlation functions since we see them as most significant for the structure of the phases. We give first a global evaluation of the correlation function patterns for a wide set of frustration ratios and then focus on a number of points to see the dependence on the guiding wave function and to deduce the trends. The paper closes with a discussion and a comparison with other results in the literature.

\section{HAMILTONIAN}

The Hamiltonian of the system refers to spins on a square lattice:

$$
\mathcal{H}=J_{1} \sum_{(i, j)} \mathbf{S}_{i} \cdot \mathbf{S}_{j}+J_{2} \sum_{[i, j]} \mathbf{S}_{i} \cdot \mathbf{S}_{j}
$$

The $\mathbf{S}_{i}$ are spin- $\frac{1}{2}$ operators and the sum is over pairs of nearest neigbors $(i, j)$ and over pairs of next-nearest neighbors $[i, j]$ on a quadratic lattice. Both coupling constants $J_{1}$ and $J_{2}$ are taken as positive. So the two types of interactions frustrate each other.

In order to prepare for the representation of the Hamiltonian we express the spin components in spin raising and lowering operators

$$
\mathbf{S}_{i} \cdot \mathbf{S}_{j}=\frac{1}{2}\left(S_{i}^{+} S_{j}^{-}+S_{i}^{-} S_{j}^{+}\right)+S_{i}^{z} S_{j}^{z} .
$$

We will use the $z$ component representation of the spins and a complete state of the spins will be represented as

$$
|R\rangle=\left|s_{1}, s_{2}, \ldots, s_{N}\right\rangle,
$$

where the $s_{j}$ are eigenvalues of the $S_{j}^{z}$ operator. The diagonal matrix elements of the Hamiltonian are in the representation (3) given by

$$
\langle R|\mathcal{H}| R\rangle=J_{1} \sum_{(i, j)} s_{i} s_{j}+J_{2} \sum_{[i, j]} s_{i} s_{j}
$$

The off-diagonal elements are between two nearby configurations $R^{\prime}$ and $R$. Here $R^{\prime}$ is the same as $R$ except at a pair of nearest-neighbors sites $(i, j)$ or next-nearest-neighbor sites $[i, j]$, for which the spins $s_{i}$ and $s_{j}$ are opposite. In $R^{\prime}$ the pair is turned over by the Hamiltonian. Then

$$
\left\langle R^{\prime}|\mathcal{H}| R\right\rangle=\frac{1}{2} J_{1} \quad \text { or } \quad\left\langle R^{\prime}|\mathcal{H}| R\right\rangle=\frac{1}{2} J_{2}
$$

depending on whether a nearest or a next-nearest pair is flipped.

\section{DMRG PROCEDURE AND ITS RESULTS}

The DMRG procedure approximates the ground-state wave function by searching through various representations in bases of a given dimension $m .{ }^{1}$ In its standard form ${ }^{13}$ the system is mapped on a one-dimensional chain (see Fig. 1)

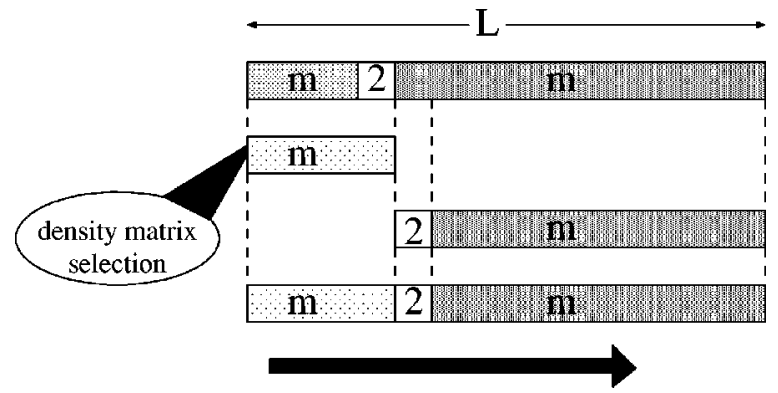

FIG. 1. The DMRG procedure with one connecting site.

and separated into two parts: a left and right hand part, connected by one (or more) site(s). The wave function is iteratively improved at the left hand side by using a basis for the right hand side and vice versa. By separating off a site from the right and including it to the left the wave function is rewritten in a new basis which is optimized by chosing the largest eigenvalues of the density matrix. Zipping back and forth until convergence to a steady state, a wave function is reached which is optimal for the given basis.

In Fig. 2 we have drawn two possible ways to map the system on a one-dimensional chain. One sees that if we divide again the chain into a left hand part and a right hand part and a connecting site, quite a few sites of the left hand part are nearest or next-nearest neighbors of sites of the right hand part. So the coupling between the two parts of the chain is not only through the connecting site but also through sites which are relatively far away from each other in the onedimensional path. The operators for the spins on these sites are not as well represented as those of the connecting site, which is fully represented by the two possible spin states. Yet the correlations between the interacting sites count as much for the energy of the system as those interacting with the connecting site. One may say that the farther away two interacting sites are in the one-dimensional chain, the poorer their influence is accounted for. This consideration explains in part why open systems can be calculated more accurately than closed systems, even in one-dimensional systems.

It is an open question which map of the two-dimensional onto a one-dimensional chain gives the best representation of the ground state of the system. We have restricted our calculations to the two paths shown here. The second choice, the " "meandering" path, was motivated by the fact that it has the strongest correlated sites most nearby in the chain. Indeed, for a given dimension $m$ of the representation, the "meandering" path gives a lower energy than the "straight" path.

The DMRG calculations as well as the corresponding GFMC simulations are carried out for both paths. Although

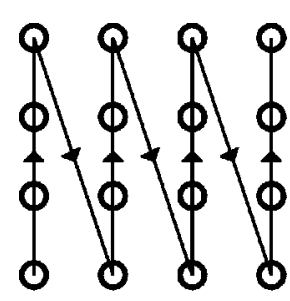

(a)

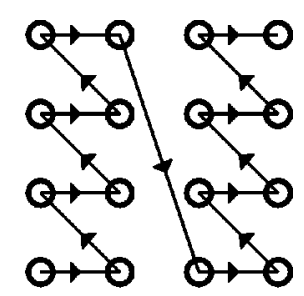

(b)
FIG. 2. Two one-dimensional paths through the system: "straight", (a) and "meandering", (b). 


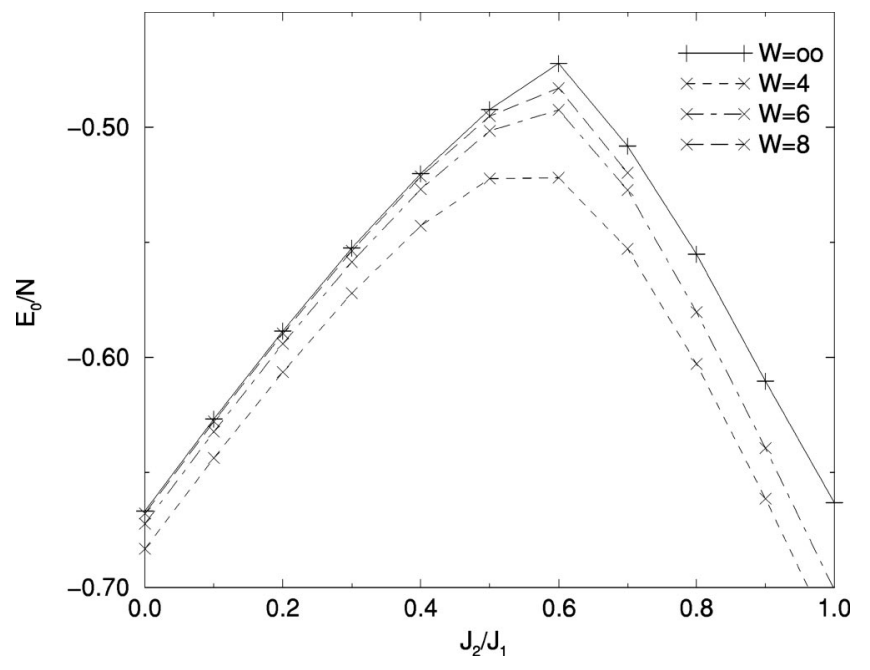

FIG. 3. The energy as function of the frustration ratio.

the meandering path has to be preferred over the straight path, we have also investigated the latter, since the path chosen leaves its imprints on the resulting correlation pattern and the paths break the symmetries in different ways. Both paths have an orientational preference. In open systems the translational symmetry is broken anyway, but the meandering path has in addition a staggering in the horizontal direction. This together with the horizontal nearest-neighbor sites appearing in the meandering path gives a preference for horizontal dimerlike correlations in this path. On the other hand, the straight path prefers dimers in the vertical direction. Comparing the results of the two choices allows us to draw further conclusions on the nature of the intermediate state.

We now give a brief summary of the results of a pure DMRG calculation. Extensive details can be found in Ref. 14. The system consists of strips of widths up to $W=8$ and of various lengths $L$. They are periodic in the small direction and open in the long direction. The periodicity enables us to study the spin stiffness. We have chosen open boundaries in the long direction to avoid the errors in the DMRG wave function due to periodic boundaries. Since we have good control of the scaling behavior in $L$, we extrapolate to $L$ $\rightarrow \infty .{ }^{14}$ In the small direction we are restricted to $W=2,4,6$, and 8 as odd values are not compatible with the antiferromagnetic character of the system. For wider system sizes the number of states which has to be taken into account exceeds the possiblities of the present workstations. Our criterion is that the value of the energy not drift anymore appreciably upon the inclusion of more states. This does not mean that the wave function is virtually exact, since the energy is a rather insensitive probe for the wave function. For instance correlation functions still improve from the inclusion of more states.

In Fig. 3 we present the energy as function of the ratio $J_{2} / J_{1}$, for strip widths 4,6 , and 8 together with the best extrapolation to infinite-width systems. The figure strongly suggests that the infinite system undergoes a first-order phase transition around a value 0.6. This can be attributed to the transition to a columnar order (lines of opposite magnetization). It is impossible to deduce more information from such an energy curve as other phase transitions are likely to be

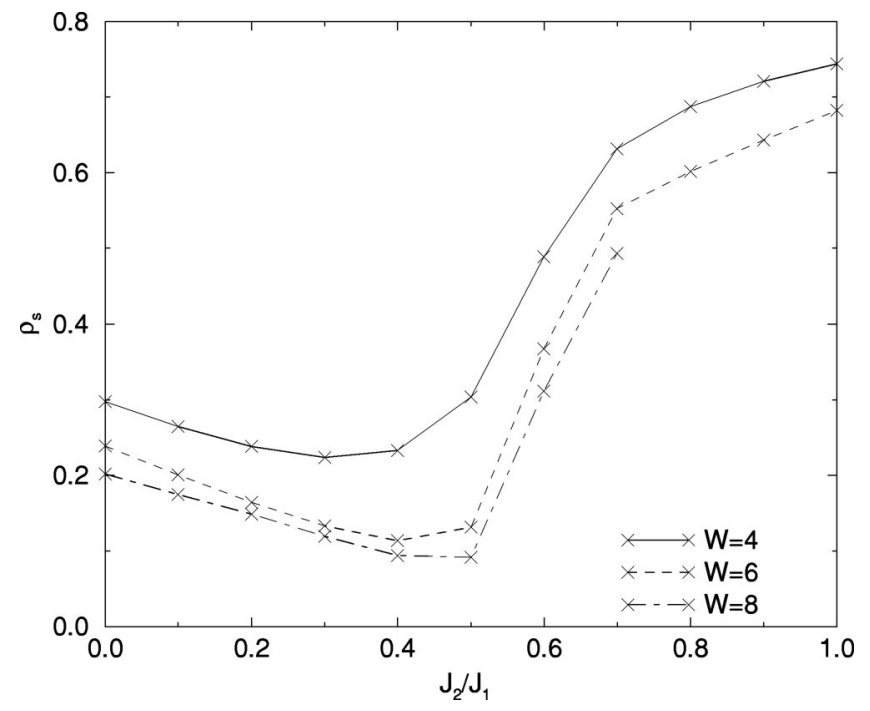

FIG. 4. The stiffness $\rho_{s}$ as function of the frustration ratio. Finite-size extrapolations put the region where $\rho_{s}$ vanishes between 0.38 and 0.62 (see Ref. 7).

continuous with small differences in energy between the phases.

The spin stiffness can be calculated with the DMRG wave function for systems which are periodic in at least one direction. ${ }^{14}$ For the formula used we refer to the expression given in Ref. 7.

The result of the computation is plotted in Fig. 4. One observes a substantial decrease of $\rho_{s}$ in the frustrated region indicating the appearance of a magnetically disordered phase. In contrast to the energy the data do not allow a meaningful extrapolation to large widths. The lack of clear finitesize scaling behavior in the regime of small values of $W$ prevents us from drawing firm conclusions on the disappearence of the stiffness in the middle regime.

For the correlation functions following from the DMRG wave function we refer to Ref. 14 .

\section{EXTRACTING CONFIGURATIONS FROM THE DMRG WAVE FUNCTION}

In order to prepare for the use of DMRG wave functions in a GFMC simulation we analyze the structure of the DMRG process. The central quantities are the partial density matrices. The configurations of the right hand part and the left hand part are denoted by $R_{r}$ and $R_{l}$. Then the density matrix for the left hand part reads

$$
\left\langle R_{l}|\rho| R_{l}^{\prime}\right\rangle=\sum_{R_{r}}\left\langle R_{l}, R_{r} \mid \Phi\right\rangle\left\langle\Phi \mid R_{l}^{\prime}, R_{r}\right\rangle .
$$

White ${ }^{1}$ has shown that the best way to represent the state $|\Phi\rangle$ is to select the $m$ eigenstates $|\alpha\rangle$ with the largest eigenvalue

$$
\sum_{R_{l}^{\prime}}\left\langle R_{l}|\rho| R_{l}^{\prime}\right\rangle\left\langle R_{l}^{\prime} \mid \alpha\right\rangle=\lambda_{\alpha}\left\langle R_{l} \mid \alpha\right\rangle .
$$

In practice we do not solve the eigenvalues of the density matrix in the configuration representation, but in a projection on a smaller basis. 
The first step is the analysis of a set of representations for the wave function in terms of two parts (without a connecting site in between). Let the left hand part contain $l$ sites and the other part $N-l$ sites. We denote the $m$ basis states of the left hand part by the index $\alpha$ and those of the right hand part by $\bar{\alpha}$. The eigenstates of the two parts are closely linked and related as follows:

$$
\begin{aligned}
& \left\langle R_{l} \mid \alpha\right\rangle=\frac{1}{\sqrt{\lambda_{\alpha}}} \sum_{R_{r}}\left\langle\Phi \mid R_{l}, R_{r}\right\rangle\left\langle R_{r} \mid \bar{\alpha}\right\rangle, \\
& \left\langle R_{r} \mid \bar{\alpha}\right\rangle=\frac{1}{\sqrt{\lambda_{\alpha}}} \sum_{R_{l}}\left\langle\alpha \mid R_{l}\right\rangle\left\langle R_{l}, R_{r} \mid \Phi\right\rangle .
\end{aligned}
$$

It means that for every eigenvalue $\lambda_{\alpha}$ there is an eigenstate $\alpha$ for the left hand part and an $\bar{\alpha}$ for the right hand density matrix. The proof of Eqs. (8) follows from insertion in the density matrix eigenvalue equation (7).

The second step is an expression for the ground-state wave function in terms of these eigenfunctions. Generally we have

$$
\left\langle R_{l}, R_{r} \mid \Phi\right\rangle=\sum_{\alpha, \bar{\beta}}\left\langle R_{l} \mid \alpha\right\rangle\left\langle R_{r} \mid \bar{\beta}\right\rangle\langle\alpha \bar{\beta} \mid \Phi\rangle,
$$

while due to Eqs. (8) we find

$$
\begin{aligned}
\langle\alpha \bar{\beta} \mid \Phi\rangle & =\sum_{R_{l}, R_{r}}\left\langle\alpha \mid R_{l}\right\rangle\left\langle\bar{\beta} \mid R_{r}\right\rangle\left\langle R_{l}, R_{r} \mid \Phi\right\rangle \\
& =\sqrt{\lambda_{\alpha}} \sum_{R_{r}}\left\langle\bar{\beta} \mid R_{r}\right\rangle\left\langle R_{r} \mid \bar{\alpha}\right\rangle=\delta_{\alpha, \beta} \sqrt{\lambda_{\alpha}} .
\end{aligned}
$$

Thus we can represent the ground state as

$$
\left\langle R_{l}, R_{r} \mid \Phi\right\rangle=\sum_{\alpha} \sqrt{\lambda_{\alpha}^{l}}\left\langle R_{l} \mid \alpha\right\rangle_{l}\left\langle R_{r} \mid \bar{\alpha}\right\rangle_{N-l}
$$

This representation is used for calculating the wave function in a configuration $R$. As in the proces of constructing the DMRG wave function the density matrices are diagonalized all the time it is easy to make a table of the $m$ eigenvalues $\lambda_{\alpha}^{l}$ for each division $l$. The corresponding eigenfunctions, however, cannot be stored (too many configurations) and so the next step is to find an algorithm for these functions.

As intermediary we consider a representation of the wave function with one site $s_{l}$ separating the spins $s_{1} \cdots s_{l-1}$ on the left hand side from $s_{l+1} \cdots s_{N}$ on the right hand side. Using the same basis as in Eq. (11) we have

$$
\begin{aligned}
\left\langle s_{1} \cdots s_{l-1}, s_{l}, s_{l+1} \cdots s_{N} \mid \Phi\right\rangle= & \sum_{\alpha, \alpha^{\prime}}\left\langle s_{1} \cdots s_{l-1} \mid \alpha\right\rangle \phi_{\alpha, \alpha^{\prime}}^{l}\left(s_{l}\right) \\
& \times\left\langle s_{l+1} \cdots s_{N} \mid \bar{\alpha}^{\prime}\right\rangle
\end{aligned}
$$

We compare this representation in two ways with Eq. (11). First we combine the middle site with the left hand part. This leads to $m$ states which can be expressed as linear combinations of the states of the enlarged segment

$$
\sum_{\alpha}\left\langle s_{1} \cdots s_{l-1} \mid \alpha\right\rangle \phi_{\alpha, \alpha^{\prime}}^{l}\left(s_{l}\right)=\sum_{\alpha^{\prime \prime}}\left\langle s_{1} \cdots s_{l} \mid \alpha^{\prime \prime}\right\rangle T_{\alpha^{\prime \prime}, \alpha^{\prime}}^{l}
$$

In fact this relation is the very essence of the DMRG procedure. The wave function in the larger space is projected onto the eigenstates of the the density matrix of that space. Since the process of zipping back forth has converged, there is indeed a fixed relation (13). However, when we insert Eq. (13) into Eq. (12) and compare it with Eq. (11) we conclude that the matrix $T$ must be diagonal:

$$
T_{\alpha^{\prime \prime}, \alpha^{\prime}}^{l}=\delta_{\alpha^{\prime \prime}, \alpha^{\prime}} \sqrt{\lambda_{\alpha^{\prime}}^{l}}
$$

This leads to the recursion relation

$$
\left\langle s_{1} \cdots s_{l} \mid \alpha^{\prime}\right\rangle=\sum_{\alpha}\left\langle s_{1} \cdots s_{l-1} \mid \alpha\right\rangle A_{\alpha, \alpha^{\prime}}^{l}\left(s_{l}\right),
$$

with

$$
A_{\alpha, \alpha^{\prime}}^{l}\left(s_{l}\right)=\phi_{\alpha, \alpha^{\prime}}^{l}\left(s_{l}\right) \sqrt{\lambda_{\alpha^{\prime}}^{l}} .
$$

We extract and store the $A$ matrices for all divisions from the construction of the DMRG wave function. With the recursion relation (15) we can calculate the left hand part of the wave function in any configuration.

The second combination concerns the contraction of the middle site with the right hand part. This leads to the recursion relation

$$
\left\langle s_{l} \cdots s_{N} \mid \bar{\alpha}\right\rangle=\sum_{\alpha^{\prime}} B_{\alpha, \alpha^{\prime}}^{l-1}\left(s_{l}\right)\left\langle s_{l+1} \cdots s_{N} \mid \bar{\alpha}^{\prime}\right\rangle
$$

with

$$
B_{\alpha, \alpha^{\prime}}^{l-1}(s)=\phi_{\alpha, \alpha^{\prime}}^{l}(s) \sqrt{\lambda_{\alpha}^{l-1}} .
$$

This allows us to calculate recursively the right hand part of the wave function for any configuration from the $B$ matrices. Thus the $A$ and $B$ matrices are the essential ingredients of the calculation of the wave function. From Eqs. (18) and (16) follows that they are related as

$$
B_{\alpha, \alpha^{\prime}}^{l-1}(s)=\sqrt{\lambda_{\alpha^{\prime}}^{l} / \lambda_{\alpha}^{l-1}} A_{\alpha, \alpha^{\prime}}^{l}(s) .
$$

The value of the wave function is obtained as the product of matrices acting on a vector. Thus the calculational effort scales with $\mathrm{m}^{2}$. Using relation (19) one reconfirms by direct calculation that the wave function is indeed independent of the division $l$.

When the simulation is in the configuration $R$, all the $\left\langle R_{l} \mid \alpha\right\rangle_{l}$ and the $\left\langle R_{r} \mid \bar{\alpha}\right\rangle_{N-l}$ are calculated and stored, with the purpose of calculating the wave functions more efficiently for the configurations $R^{\prime}$ which are connected to $R$ by the Hamiltonian and which are candidates for a move. The structure of these nearby states is $R^{\prime}=s_{1} \cdots s_{l_{2}} \cdots s_{l_{1}} \cdots s_{N}\left(l_{2}\right.$ $\left.>l_{1}\right)$. So we have that for $R^{\prime}$ the representation

$$
\left\langle R^{\prime} \mid \Phi\right\rangle=\sum_{\alpha} \sqrt{\lambda_{\alpha}^{l_{2}}}\left\langle s_{1} \cdots s_{l_{2}} \cdots s_{l_{1}} \mid \alpha\right\rangle\left\langle s_{l_{2}+1} \cdots s_{N} \mid \bar{\alpha}\right\rangle
$$


holds. Now we see the advantage of having the wave function stored for all the divisions. The second factor in Eq. (20) is already tabulated; the first factor involves a number of matrix multiplications equal to the distance in the chain of the two spins $l_{1}$ and $l_{2}$ until one reaches a tabulated function. One can use the tables for a certain number of moves but after a while it starts to pay off to make a fresh list.

In summary we have the following three steps in the procedure.

(i) The ground-state wave function is constructed as indicated in the previous section.

(ii) From this ground-state wave function the eigenvalues $\lambda_{\alpha}^{l}$ and the matrices $A_{\alpha, \alpha^{\prime}}^{l}(s)$ and $B_{\alpha, \alpha^{\prime}}^{l}(s)$ are derived as described in this section. They are stored for use in the simulation.

(iii) For a given configuration $R$ tables of inner products $\left\langle R_{l} \mid \alpha\right\rangle_{l}$ and $\left\langle R_{r} \mid \bar{\alpha}\right\rangle_{N-l}$ are constructed and stored.

The first two steps have to be carried out only once; the last one has to be performed regularly in the simulation process.

\section{RESULTS FOR GFMC SIMULATIONS WITH SR}

We now come to the crux of this study: the simulations of the system with the GFMC method, using the DMRG wave functions to guide the importance sampling. We use the fixed node technique introduced by Ceperley and Alder, ${ }^{12}$ adapted to the lattice by ten Haaf van Bemmel and co-workers ${ }^{15,16}$ and augmented by stochastic reconfiguration by Sorella. ${ }^{3}$ The procedure is comprehensively and lucidly described by Sorella and Capriotti ${ }^{17}$ to which we refer for details. We use a continuous "time" $\epsilon$ and an improved (mixed) estimator ${ }^{18}$ for the correlation functions. While the estimates for the energy are "exact" in a correct simulation, independent of the guiding wave function, the mixed estimator for the correlation functions is limited by the quality of the guiding wave function. All the simulations have been carried out for a 10 $\times 10$ lattice with open boundaries. Standardly we have 6000 walkers and we run the simulations for about $10^{4}$ measurements. These measuring points are not fully independent and the variance is determined by chopping up the simulations into 50-100 groups. Since various MC runs are independent, they could be carried out simultaneously on a cluster of computers.

Open boundary conditions have the disadvantage of boundary effects, which make it more difficult to distinguish between spontaneous and induced breaking of the translational symmetry. On the other hand, for open boundaries, dimers, plaquettes, or any other interruption of the translational symmetry have a natural reference frame. The correlations are not only influenced by the boundaries of the system; also the guiding DMRG wave function leaves its imprint on the results. This is mainly due to the fact that we have only mixed estimators for the correlation functions, which show a mix of the guiding wave function and the true wave function. The improved estimator, used in these pictures, corrects for this effect to linear order in the deviation. The ladderlike structure in the DMRG path is reflected in a ladderlike pattern in the correlations as an inspection of the correlations in the DMRG wave functions (not shown here)
TABLE I. For each degree of frustration the imaginary time interval $\epsilon$, the energy of the guiding state $E_{\mathrm{DMRG}}$, and the energy of the GFMC state $E_{\mathrm{GFMC}}$ are listed.

\begin{tabular}{llcccc}
\hline \hline & \multicolumn{2}{c}{ Straight } & \multicolumn{2}{c}{ Meander } \\
$J_{2}$ & $\epsilon$ & $E_{\mathrm{DMRG}}$ & $E_{\mathrm{GFMC}}$ & $E_{\mathrm{DMRG}}$ & $E_{\mathrm{GFMC}}$ \\
\hline 0.0 & 0.3 & -61.30 & $-62.33(8)$ & -61.84 & $-62.54(4)$ \\
0.1 & 0.06 & -57.96 & & -58.53 & $-59.25(2)$ \\
0.2 & 0.04 & -54.75 & $-56.08(11)$ & -55.48 & $-56.22(4)$ \\
0.3 & 0.02 & -51.75 & $-53.17(4)$ & -52.50 & $-53.38(3)$ \\
0.4 & 0.02 & -49.00 & $-50.51(8)$ & -49.92 & $-50.60(5)$ \\
0.5 & 0.014 & -46.68 & $-47.76(6)$ & -47.78 & $-48.34(4)$ \\
0.6 & 0.015 & -45.41 & & -46.03 & $-46.40(3)$ \\
0.7 & 0.015 & -45.67 & & -45.60 & $-46.00(2)$ \\
0.8 & 0.02 & -49.16 & & -49.13 & $-49.60(9)$ \\
0.9 & 0.02 & -53.61 & & -53.70 & $-54.52(2)$ \\
1.0 & 0.02 & -58.46 & $-59.71(9)$ & -58.64 & $-59.80(8)$ \\
\hline \hline
\end{tabular}

reveals. But ladders are clearly also present in the GFMC results shown in the pictures.

We first give an overall assessment of the correlation function pattern and then analyze some values of the ratio $J_{2} / J_{1}$. In the first series we have used the guiding wave function on the basis of the meandering path, Fig. 2(b), because it gives a better energy than the straight option (a). The number of basis states is $m=75$, which is small enough to carry out simulations with reasonable speed and large enough that trends begin to manifest themselves. Measurements of a number of correlation functions are made in conjunction with stochastic reconfiguration (see Ref. 17). The details of these calculations are given in Table I. Note that the DMRG guiding wave function gives a better energy for the meandering path than for the straight path for values of $J_{2} / J_{1}$ up to 0.6 . From 0.7 on, this difference is virtually absent. This undoubtly has to do with the change to the columnar state which can equally well be realized by both paths. The value of $\epsilon$ has been chosen as a compromise: independent measurements require a large $\epsilon$ but the minus sign problem requires one to apply often stochastic reconfiguration, i.e., a small $\epsilon$. One sees that in the heavily frustrated region the $\epsilon$ must be taken as small. In fact more detailed calculations for $J_{2}=0.3 J_{1}$ and $J_{2}=0.5 J_{1}$ were carried out with $\epsilon=0.01$.

In Figs. 6 and 7 we have plotted a sequence of visualizations of the correlations. From top to bottom (zig-zag) they give correlations for the values of $J_{2} / J_{1}$. In order to highlight the differences a distinction is made between correlations which are above average (solid lines) and below average (dashed lines). All nearest-neighbor spin correlations shown are negative. In all the pictures one sees the influence of the boundaries on the spin correlations. Only 1/4 of the lattice has been pictured; the other segments follow by symmetry. The upper right corner, which corresponds to the center of the lattice, is the most significant for the behavior of the bulk. The overall trend is that spatial variations in the correlation functions occur in growing size with $J_{2} / J_{1}$. On the side of low $J_{1} / J_{2}$ (Néel phase) one sees dimer patterns in the horizontal direction; they turn over to vertical dimers (around $J_{2}=0.7 J_{1}$ ) and rapidly disappear in the columnar 
TABLE II. Interpolation (a) and extrapolation (b) estimates of the energy per site of a $10 \times 10$ lattice.

(a)

\begin{tabular}{cl}
$L$ & $e_{0}(L \times L)$ \\
\hline 4 & -0.5740 \\
6 & -0.6031 \\
8 & -0.6188 \\
10 & $-0.629(1)$ \\
$\infty$ & $-0.669437(5)$
\end{tabular}

(b)

\begin{tabular}{cccc} 
No. states & Trunc. error & $e_{0}(\mathrm{DMRG})$ & $e_{0}(\mathrm{GFMC})$ \\
\hline 32 & $21.2 \times 10^{-5}$ & -0.6084 & $-0.6192(1)$ \\
75 & $12.0 \times 10^{-5}$ & -0.6184 & $-0.6254(5)$ \\
100 & $10.5 \times 10^{-5}$ & -0.6201 & $-0.625(2)$ \\
128 & $8.7 \times 10^{-5}$ & -0.6214 & $-0.6269(6)$ \\
150 & $9.6 \times 10^{-5}$ & -0.6231 & $-0.6277(5)$ \\
$2^{N}$ & 0 & $-0.631(3)$ & \\
\hline \hline
\end{tabular}

phase. This is again support for the fact that the columnar phase is separated from the intermediate state by a first-order phase transition.

In order to eliminate the influence of the guiding wave function we scrutinize some of values of $J_{2} / J_{1}$ in more detail by inspecting how the results depend on the size of the basis in the DMRG wave function and on the choice of the DMRG path. Since we are mostly interested in the behavior of the infinite lattice, we discuss mainly the behavior of the correlations in and around the central plaquette. So we study a sequence of DMRG wave functions for $m=32,75,100$, 128, and 150(200) and carry out for each of them extensive GFMC simulations. First we look to the case $J_{2}=0$, which is easy because it is nonfrustrated ${ }^{19}$ and we know that it must be Néel ordered and therefore it serves as a check on the calculations. Then we take $J_{2}=0.3 J_{1}$ which is the most difficult case since it is likely to be close to a phase transition. Finally we inspect $J_{2}=0.5 J_{1}$ where we are fairly sure that some dimerlike phase is realized.

$$
\text { A. } J_{2}=0
$$

For the unfrustrated Heisenberg model we have several checkpoints for our calculations. We can find to a high degree of accuracy the ground-state energy and we are sure that the Néel phase is homogeneous, i.e., that the correlations show no spatial variation other than that of the antiferromagnet. We have two ways of estimating the energy of a 10 $\times 10$ lattice. The first method is based on finite-size interpolation. From DMRG calculations ${ }^{14}$ we have an exact value for a $4 \times 4$ lattice, an accurate value for the $6 \times 6$ lattice, and a good value for the $8 \times 8$ lattice. There is also the very accurate calculation of Sandvik ${ }^{4}$ for an infinitely large lattice, yielding the value of $e_{0}=-0.669437(5)$. The leading finitesize correction goes as $1 / L$. Including also a $1 / L^{2}$ term we have esimated the value for a $10 \times 10$ lattice as $0.629(1)$ and incorporated this value in Table II(a). We stress that this is an interpolation for which the value of Sandvik is the most important input.

The second method is less well founded and uses the experience that DMRG energy estimates can be improved considerably by extrapolating to zero truncation error. When plotted as function of this truncation error the energy is often remarkably linear. In Table II(b) we give for a series of bases $m=32,75,100,128$, and 150 , the values of the truncation error and the corresponding DMRG energy per site together with the extrapolation on the basis of linear behavior. ${ }^{20}$ Note that the two estimates are compatible. In Table II(b) we have also listed the values of the GFMC simulations for the corresponding values of $m$. They do agree quite well with these estimates, in particular with the one based on finite-size scaling. We point out that one would have to go very far in the number of states in the DMRG calculation to obtain an accuracy that is easily obtained with GFMC simulations. Thus the combination of GFMC and DMRG techniques does really better than the individual components. One might wonder why there is still a drift to lower energy values in the GFMC simulations (which is also present in the tables to come). The reason is that the DMRG wave function is strictly zero outside a certain domain of configurations, because the truncation of the basis involves also the elimination of certain combinations of conserved quantities of the constituing parts. The domain of the wave function grows with the size of the basis.

Turning now to the correlations it seems that they are homogeneous in the center of the lattice for $J_{2}=0$. However, a closer inspection reveals small differences. In Table III we list the asymmetries in the horizontal and vertical directions of the spin correlations in and around the central plaquette as a function of the number of states. If we number the spins on the lattice as $\mathbf{S}_{n, m}$ with $1 \leqslant n, m \leqslant 10$, the central plaquette has the coordinates $(5,5),(5,6),(6,5)$, and $(6,6)$. We then define the asymmetry parameters $\Delta_{x}$ and $\Delta_{y}$ as

TABLE III. Values for the asymmetry in the center for $J_{2}=0$. As discussed in the text the error in the improved estimator values is of the order of 0.02 , which means that for $m=128$ and higher the values are statistically indistinguishable from zero.

\begin{tabular}{cccccrr}
\hline \hline No. states & \multicolumn{3}{c}{$\Delta_{x}$} & & \multicolumn{2}{c}{$\Delta_{y}$} \\
\hline$m$ & DMRG & GFMC & Improved & \multicolumn{1}{c}{ DMRG } & GFMC & Improved \\
32 & 0.14373 & 0.09981 & 0.056 & -0.00060 & 0.00078 & 0.00216 \\
75 & 0.07291 & 0.05668 & 0.040 & 0.00081 & 0.00601 & 0.01121 \\
100 & 0.06432 & 0.04255 & 0.031 & 0.00030 & 0.00173 & 0.00316 \\
128 & 0.05619 & 0.03734 & 0.018 & 0.00091 & -0.00040 & -0.00173 \\
150 & 0.05044 & 0.03612 & 0.022 & 0.00079 & 0.00261 & 0.00442 \\
\hline \hline
\end{tabular}


TABLE IV. Energies and asymmetries for the case $J_{2}=0.3 J_{1}$ as function of the number of basis states $m$. Here $\delta$ is the truncation error. The asymmetries $\Delta_{x}$ and $\Delta_{y}$ for the GFMC simulations are calculated with the improved estimator. The guiding wave function is obtained from the meandering path (b) in Fig. 2. The statistical error in $\Delta_{x}$ and $\Delta_{y}$ is of the order of 0.02 .

\begin{tabular}{crrcccrr}
\hline \hline \multirow{2}{*}{$\begin{array}{c}\text { No. states } \\
m\end{array}$} & \multicolumn{9}{c}{ DMRG } & \multicolumn{3}{c}{ GFMC } \\
& $\delta^{*} 10^{5}$ & $E_{\text {DMRG }}$ & $\Delta_{x}$ & $\Delta_{y}$ & $E_{\text {GFMC }}$ & $\Delta_{x}$ & $\Delta_{y}$ \\
\hline 32 & 19.0 & -51.609 & 0.27784 & 0.00295 & $-52.81(43)$ & 0.363 & -0.009 \\
75 & 10.6 & -52.581 & 0.15462 & 0.00616 & $-53.29(05)$ & 0.207 & 0.011 \\
100 & 9.4 & -52.707 & 0.14709 & 0.00943 & $-53.32(33)$ & 0.145 & 0.009 \\
128 & 10.6 & -52.821 & 0.13042 & 0.00577 & $-54.01(04)$ & 0.254 & 0.063 \\
150 & 10.4 & -52.888 & 0.12564 & 0.00737 & $-54.10(12)$ & 0.236 & 0.103 \\
\hline \hline
\end{tabular}

$$
\begin{aligned}
\Delta_{x}= & \frac{1}{4}\left\langle\mathbf{S}_{4,5} \cdot \mathbf{S}_{5,5}+\mathbf{S}_{4,6} \cdot \mathbf{S}_{5,6}+\mathbf{S}_{6,5} \cdot \mathbf{S}_{7,5}+\mathbf{S}_{6,6} \cdot \mathbf{S}_{7,6}\right\rangle \\
& -\frac{1}{2}\left\langle\mathbf{S}_{5,5} \cdot \mathbf{S}_{6,5}+\mathbf{S}_{5,6} \cdot \mathbf{S}_{6,6}\right\rangle, \\
\Delta_{y}= & \frac{1}{4}\left\langle\mathbf{S}_{5,4} \cdot \mathbf{S}_{5,5}+\mathbf{S}_{6,4} \cdot \mathbf{S}_{6,5}+\mathbf{S}_{5,6} \cdot \mathbf{S}_{5,7}+\mathbf{S}_{6,6} \cdot \mathbf{S}_{6,7}\right\rangle \\
& -\frac{1}{2}\left\langle\mathbf{S}_{5,5} \cdot \mathbf{S}_{5,6}+\mathbf{S}_{6,5} \cdot \mathbf{S}_{6,6}\right\rangle .
\end{aligned}
$$

So $\Delta_{x}$ is the average value of the correlations on the four horizontal bonds which are connected to the central plaquette minus the average of the values on the two horizontal bonds in the plaquette. Similarly $\Delta_{y}$ corresponds to the vertical direction. The values for the asymmetry in Table III in the vertical direction are so small that they have no significance. Note that the anticipated decrease in $\Delta_{x}$ is slow in the DMRG method and therefore also slow in the mixed estimator of the GFMC simulations. The improved estimator, however, is truely an improvement. So one sees that all observed small deviations from the homogeneous state will disappear with the increase of the number of states in the basis of the DMRG wave function. (In general the accuracy of the correlations is determined by that of the GFMC simulations. We get as variance a number of the order 0.01 , implying twice that value for the improved estimator.) The vanishing of $\Delta_{x}$ and $\Delta_{y}$ also proves that finite-size effects are small in the center of the $10 \times 10$ lattice. From these data we may conclude that the GFMC simulations can make up for the errors in the DMRG wave function for a relative low number of basis states. We have not carried out a similar series for the straight path since this will certainly show no dimers as will become clear from the following cases.

$$
\text { B. } J_{2}=0.3 J_{1}
$$

This case is the most difficult to analyze since it is expected to be close to a continuous phase transition from the Néel state to a dimerlike state. As is known ${ }^{21}$ the DMRG structure of the wave function is not very adequate to cope with the long-range correlation in the spins typical for a critical point. In Table IV we have presented the same data as in Table III but now for $J_{2}=0.3$. There is no pattern in the energy as function of the truncation error $\delta$. The decrease of the energy as a function of the size of the basis $m$ in the DMRG wave functions is not saturated. The GFMC simulations lead to a notably lower energy and they hardly show a leveling off as a function of the basis of the guiding wave function. All these points are indicators that the DMRG wave function is rather far from convergence and that more accurate data would require a much larger basis. As far as the staggering in the correlations is concerned the values for $\Delta_{x}$ are significant, also because the simulation results generally increase the values. Those for $\Delta_{y}$ are not small enough to be considered as noise. Given the fact that most authors locate the phase transition at higher values $J_{2} \simeq 0.4 J_{1}$ we would expect both $\Delta$ 's to vanish. So either the dimerlike state is realized for values as low as $J_{2}=0.3 J_{1}$ or dimer formation already starts in the Néel state.

To get more insight into the nature of the ground state we have also carried out the same set of simulations on the straight path (a) in Fig. 2. This guiding wave function shows virtually no formation of dimers in any direction as can be observed from Table V. In spite of the fact that the trends indicated in the table have not come to convergence one may

TABLE V. Comparison of the energies and the values for the asymmetry in the center for the DMRG wave function based on the first (straight) path (a) in Fig. 2 and the associated GFMC simulation; $J_{2}$ $=0.3 J_{1}$.

\begin{tabular}{rrrrrrrr}
\hline \hline \multirow{2}{*}{$\begin{array}{c}\text { No.states } \\
m\end{array}$} & \multicolumn{3}{c}{ DMRG } & \multicolumn{3}{c}{ GFMC } \\
& $\delta^{*} 10^{5}$ & \multicolumn{1}{c}{$E_{\text {DMRG }}$} & \multicolumn{1}{c}{$\Delta_{x}$} & \multicolumn{1}{c}{$\Delta_{y}$} & $E_{\mathrm{GFMC}}$ & \multicolumn{1}{c}{$\Delta_{x}$} & $\Delta_{y}$ \\
\hline 32 & 30.0 & -50.672 & 0.00032 & 0.01657 & $-52.15(11)$ & 0.061 & 0.047 \\
75 & 18.9 & -51.733 & -0.00295 & 0.00426 & $-53.21(10)$ & -0.030 & 0.036 \\
100 & 19.9 & -52.066 & 0.00349 & 0.00492 & $-53.84(72)$ & 0.061 & 0.079 \\
128 & 24.6 & -52.302 & 0.00139 & 0.00791 & $-53.50(19)$ & 0.079 & 0.027 \\
150 & 25.7 & -52.455 & 0.00222 & 0.00780 & $-53.52(10)$ & 0.022 & 0.065 \\
\hline \hline
\end{tabular}


TABLE VI. Energies and asymmetries for $J_{2}=0.5 J_{1}$ with guiding wave function based on the meandering path (b) in Fig. 2.

\begin{tabular}{cccccccc}
\hline \hline \multirow{2}{*}{$\begin{array}{c}\text { No. states } \\
m\end{array}$} & $\delta^{*} 10^{5}$ & $E_{\mathrm{DMRG}}$ & $\Delta_{x}$ & $\Delta_{y}$ & $E_{\mathrm{GFMC}}$ & $\Delta_{x}$ & $\Delta_{y}$ \\
\hline 32 & 11.8 & -47.116 & 0.43245 & 0.14667 & $-47.55(29)$ & 0.295 & 0.065 \\
75 & 17.4 & -47.771 & 0.38954 & 0.13059 & $-48.22(04)$ & 0.339 & 0.070 \\
100 & 12.4 & -47.924 & 0.39364 & 0.07877 & $-48.37(22)$ & 0.310 & 0.110 \\
128 & 8.4 & -48.014 & 0.37317 & 0.08246 & $-48.32(05)$ & 0.336 & 0.139 \\
150 & 8.3 & -48.088 & 0.35819 & 0.07983 & $-48.33(12)$ & 0.324 & 0.112 \\
200 & 7.6 & -48.153 & 0.34590 & 0.09973 & $-48.43(05)$ & 0.272 & 0.094 \\
\hline \hline
\end{tabular}

draw a few conclusions from comparison of the two sets of simulations. The overal impression is that the meandering guiding wave function represents a ground state of a different symmetry as compared to the straight path guiding wave function. The meandering wave function prefers dimers in the horizontal direction and the straight wave function leads to some dimerization in the vertical direction. The difference also shows up in the energy; it is not only large on the DMRG level but it also persists at the GFMC level. We see similar trends in the next case.

$$
\text { C. } J_{2}=0.5 J_{1}
$$

By any estimate this value of the next-nearest-neighbor coupling leads to a dimerlike state if it exists at all. No accurate data are available on the energy of the $10 \times 10$ system to compare to our results. In Table VI we list the data for a set of DMRG wave functions with bases $m=32,75,100$, 128, 150, and 200. The DMRG values of the energy (with the exception of the value for $m=32$ ) can be extrapolated to zero truncation error with the limiting value $E_{0}=-48.4(1)$, which corresponds very well with the level in GFMC values for larger sizes of the basis. This indicates again that GFMC simulations can make up for the shortcomings of the DMRG wave function. One would indeed have to enlarge the basis to $m$ of the order of 1000 in order to achieve the value of the energy of the simulations which use DMRG guiding wave functions with a basis of the order of 100 .

The staggering in the correlations expressed by the quantities $\Delta_{x}$ for the horizontal direction and $\Delta_{y}$ for the vertical direction has values that are significant. If one looks to the contributions of the DMRG wave function and the GFMC simulations separately, one observes that the overall values do agree quite well, with the tendency that the GFMC simulations lower the staggerring in the horizontal direction and slightly increase it in the vertical direction. So we may con- clude that indeed in the ground state of the $J_{2}=0.5 J_{1}$ system, the correlations of the spins are not translation invariant but show a staggering. However, these results neither confirm the picture that the dimer state is the lowest (as suggested by Kotov et $a l .{ }^{8}$ ) nor support the scenario that the plaquette state is the ground state (as concluded by Capriotti and Sorella $a^{5}$. We comment on these discrepancies further in the discussion.

Again it is worthwhile to compare these results with a simulation on the basis of the straight path (a) in Fig. 2. Here it is manifest that the straight path prefers to have dimers in the vertical direction (Table VII). Again the impression is that the straight path leads to a different symmetry as compared to the meandering path. It is not only the different preference in the main direction of the dimers, also the secondary dimerization in the perpendicular direction, notably in the meandering case, is not present in the straight case. The fairly large difference in energy on the DMRG level becomes quite small on the GFMC level.

\section{DISCUSSION}

We have presented a method to employ the DMRG wave functions as guiding wave functions for a GFMC simulation of the ground state. Generally the combination is much better than the two individual methods. The GFMC simulations considerably improve the DMRG wave function. In the intermediate regime the properties of the GFMC simulations depend on the guiding wave function as the results for two different DMRG guiding wave functions show.

The method has been used to observe spin correlations in the frustrated Heisenberg model on a square lattice. In this discussion we focus on the intermediate region where the model is most frustrated and which is the pièce de résistance of the present research. We see patterns of strongly correlated nearest-neighbor spins, to be called dimers. To indicate

TABLE VII. Same as Table VI but now for the "straight" path, Fig. 2(a).

\begin{tabular}{ccccccrr}
\hline \hline \multirow{2}{*}{$\begin{array}{c}\text { No. states } \\
m\end{array}$} & \multicolumn{3}{c}{ DMRG } & \multicolumn{3}{c}{ GFMC } \\
\hline 32 & 69.4 & -45.756 & 0.00172 & 0.24701 & $-47.45(08)$ & 0.074 & 0.185 \\
75 & 26.2 & -46.718 & 0.00171 & 0.34950 & $-47.81(25)$ & -0.025 & 0.302 \\
100 & 21.2 & -46.993 & 0.00063 & 0.33131 & $-48.16(06)$ & -0.003 & 0.350 \\
128 & 24.6 & -47.231 & -0.00029 & 0.32994 & $-48.31(08)$ & 0.013 & 0.291 \\
150 & 25.7 & -47.379 & 0.00215 & 0.32458 & $-48.33(06)$ & -0.026 & 0.257 \\
\hline \hline
\end{tabular}




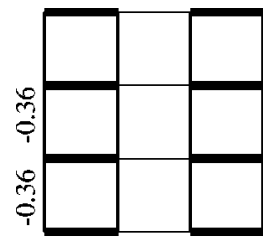

$-0.45-0.12$

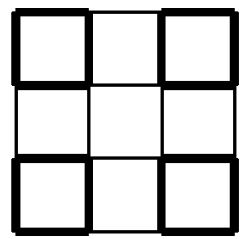

(b)

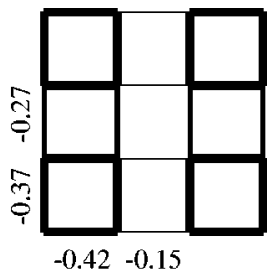

(c)
FIG. 5. The correlation pattern for the nearest spins for $J_{2}$ $=0.5 J_{1}$ : (a) according to Kotov et al. (Ref. 9), a dimer pattern in which the strength of the correlation is indicated; (b) according to Capriotti and Sorella (Ref. 5), a plaquette state; and (c) according to this paper, an intermediate pattern in which the translational invariance is broken in both directions but with unequal strength. The values indicated are those based on the meandering path and the improved estimator.

what me mean by strong and weak we give values in and around the central square of the $10 \times 10$ lattice for the case $J_{2}=0.5 J_{1}$. In Fig. $5(\mathrm{c})$ we have given the values of the central square extrapolated to an infinite lattice.

The values are based on the improved estimator and it is interesting to see the trends. The horizontal strong correla-
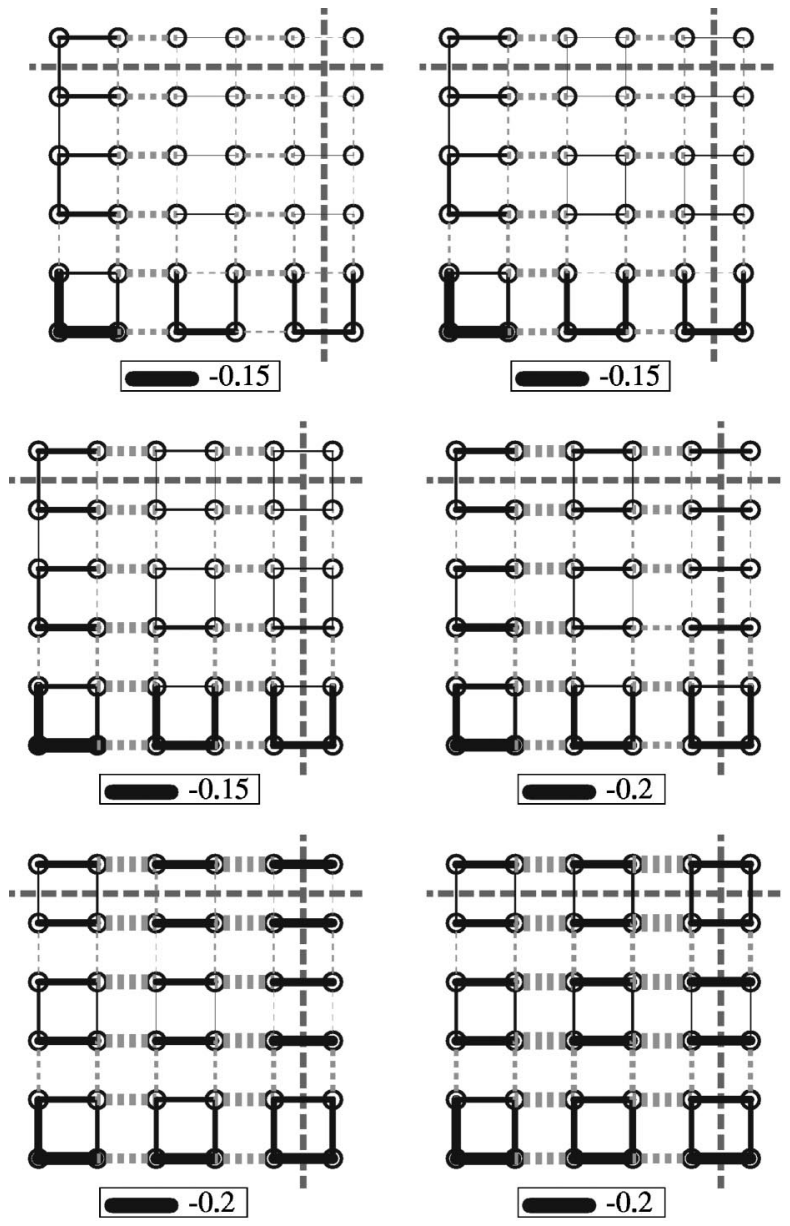

FIG. 6 . The relative correlation strengths on a $10 \times 10$ lattice. All other nearest neighbor correlations can be obtained by reflecting these pictures in the two dashed lines. The DMRG guiding state follows the meandering sequence of Fig. 2(b). More explanation is given in the text. Reading zig zag from top left to bottom right, the values for $J_{2}$ are $J_{2}=0, \ldots, 0.5$ in steps of 0.1 .
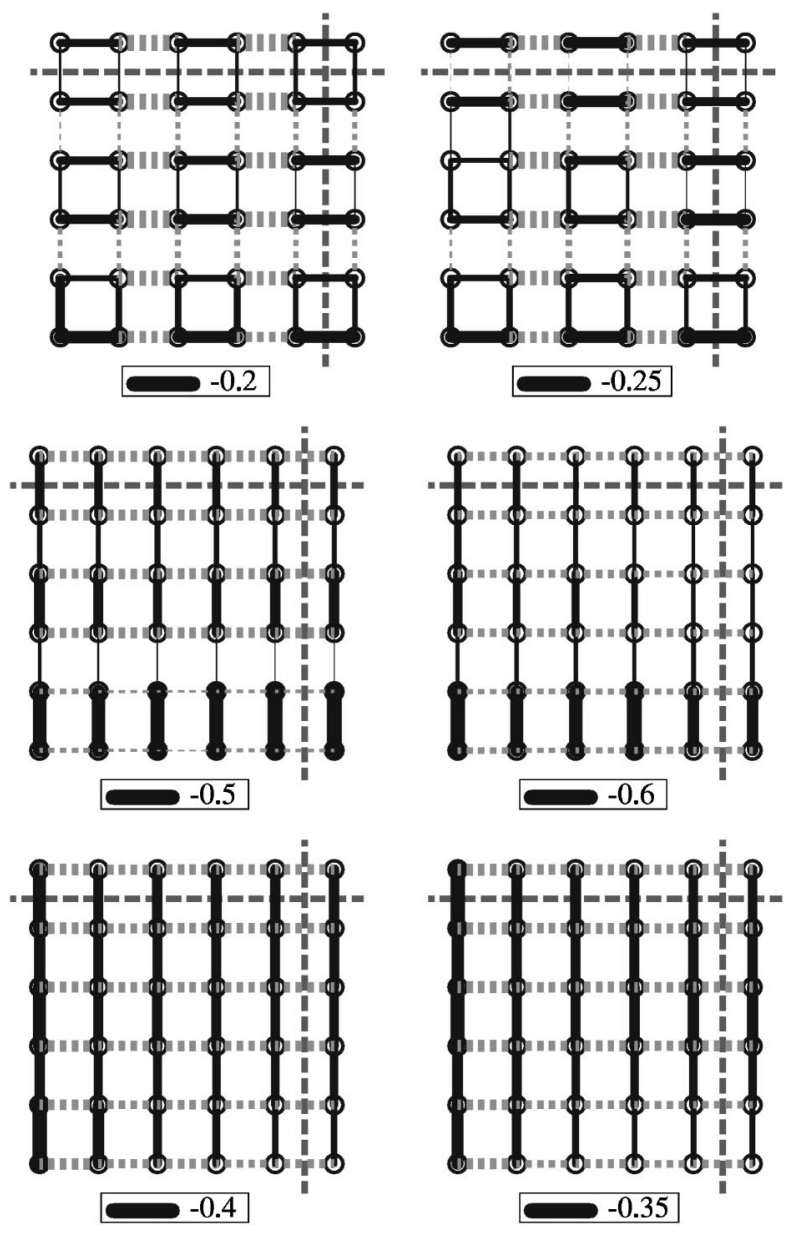

FIG. 7. The continuation of Fig. 6; the relative correlation strengths on $10 \times 10$ lattice. $J_{2}=0.5, \ldots, 1.0$ in steps of 0.1 .

tion of -0.42 is the result of the DMRG value -0.44 and the GFMC value -0.43 , while the weak bond -0.15 is the result of the DMRG value -0.09 and the GFMC value -0.12 . Thus the GFMC weakens the order parameter $D_{x}$ associated with the staggering. For the vertical direction there is hardly a change from the DMRG to GFMC value. One has to go to the next decimal to see the difference. The strong bond equals -0.368 and is coming from the DMRG value -0.375 and the GFMC value -0.371 , while the improved weak bond of -0.271 is the resulting value of -0.275 for the DMRG method and -0.273 for GFMC simulations.

Before we comment on this result we discuss the influence of the choice of the guiding wave function. We note that for both points $J_{2}=0.3 J_{1}$ and $J_{2}=0.5 J_{1}$ the two choices for the DMRG wave function give different results. First of all the main staggering is for the meandering path (b) of Fig. 2 in the horizontal direction, while the straight path (a) of Fig. 2 prefers the dimers in the vertical direction. There is not much difference in the values of the strong and weak correlations. Second, the straight path shows no appreciable staggering in the other direction, so one may wonder whether the observed effect for the meandering path is real. In our opinion this difference has to do with the effect that the DMRG wave function "locks in" on a certain symmetry. The straight path yields a ground state which is truly dimerlike in the sense that it is translational invariant in the direc- 
tion perpendicular to the dimers. The meandering path locks in on a different ground state which holds the middle between a dimerlike and a plaquettelike state. The GFMC simulations cannot overcome this difference in symmetry, likely because the two lowest states with different symmetry are virtually orthogonal. On the DMRG level there is a large difference in energy between the two states, favoring the meandering path strongly; on the GFMC level this difference has become very small. With this observation in mind we compare our result with other findings.

The results of the series expansions ${ }^{8-10}$ are shown in Fig. 5(a). Their correlations organize themselves in spin ladders. The correlations on the rungs of the ladder are $-0.45 \pm 0.5$ which compares well with our strongest horizontal correlation and this holds also for the weak horizontal correlation $(-0.12$ vs -0.15$)$. The most noticeble difference is the value of our weak correlation in the vertical direction $(-0.27$ vs -0.36$)$ while the strong correlation $(-0.37$ vs $-0.36)$ agrees. There is no real conflict between our result and theirs since the symmetry they find is fixed by the state around which the series expansion is made. So our claim is only that our state with different symmetry is the lower one. In fact in the paper of Singh et al. ${ }^{10}$ it is noted that the susceptibility to a staggering operator in the perpendicular direction (our $\Delta_{y}$ ) becomes very large in the dimer state for $J_{2}=0.5 J_{1}$ which we take as an indication of the nearby lower state. The analytical calculations in Refs. 8 and 9, however, do not support the existence of the state we find.

Neither do we find support for the plaquette state found in Ref. 5, which we have sketched in Fig. 5(b). The evidence of this investigation is based on the boundedness of the susceptibility for the operator which breaks the orientational symmetry and the divergence of the susceptibility for the order parameter breaking translational invariance (corresponding to $\Delta_{x}$ ). They have not separately investigated the values of $\Delta_{x}$ and $\Delta_{y}$ since their ground state has the symmetry of the lattice and one would find automatically the same answer. They conclude that in the absence of an orientational order parameter and with the presence of the translational order parameter the state must be plaquettelike. A scenario that reconciles this and our findings could be that starting from a fully symmetric trial function the system first breaks symmetry in a plaquettelike state and that using the plaquettelike state as the trial wave function a secondary breaking of the symmetry of the type that we find takes place.

Finally we comment on the fact that we find dimerization already for values as low as $J_{2}=0.3 J_{1}$ at least for the meandering path. As we have mentioned earlier the results as a function of the number of states have not sufficiently converged to make a firm conclusion, the more so since there is a large difference between the DMRG and GFMC techniques. Still it could be an indication that the phase transition from the Néel state to the dimer state takes place for lower values than the estimated $J_{2}=0.38 J_{1}{ }^{7}$

Thus many questions are left over, among others how the order parameters behave as function of the frustation ratio in the intermediate region. We feel that the combination of the DMRG and GFMC techniques is a good tool to investigate these issues since they demonstrate ad oculos the correlations in the intermediate state.

\section{ACKNOWLEDGMENTS}

The authors are indebted to Steve White for making his software available. One of us (M.S.L. du C. de J.) gratefully acknowledges the hospitality of Steve for a stay at Irvine of 3 months, where the basis of this work was laid. The authors have also benefited from illuminating discussions with Subir Sachdev and Jan Zaanen. The authors want to acknowledge the efficient help of Michael Patra with the simulations on the cluster of PC's of the Instituut-Lorentz. M.S.L. du C. de J. was supported by the Foundation for Fundamental Research in Matter (FOM).
${ }^{1}$ S.R. White, Phys. Rev. Lett. 69, 2863 (1992); S.R. White, Phys. Rev. B 48, 10345 (1993).

${ }^{2}$ For a recent discussion of the sign problem in various cases see $\mathrm{P}$. Henelius and A.W. Sandvik, Phys. Rev. B 62, 1102 (2000), and references therein.

${ }^{3}$ S. Sorella, Phys. Rev. Lett. 80, 4558 (1998).

${ }^{4}$ A.W. Sandvik, Phys. Rev. B 56, 11678 (1998).

${ }^{5}$ L. Capriotti and S. Sorella, Phys. Rev. Lett. 84, 3173 (2000).

${ }^{6}$ M.S.L. du Croo de Jongh and P.H.J. Denteneer, Phys. Rev. B 55, 2713 (1997).

${ }^{7}$ H.J. Schulz, T.A.L. Ziman, and D. Poilblanc, J. Phys. I 6, 675 (1996).

${ }^{8}$ V. Kotov, J. Oitmaa, O.P. Sushkov, and Z. Weihong, Phys. Rev. B 60, 14613 (1999).

${ }^{9}$ V. Kotov, J. Oitmaa, O.P. Sushkov, and Z. Weihong, Phys. Rev. B 60, 14613 (1999).

${ }^{10}$ R.R.P. Singh, Z. Weihong, C.J. Hamer, and J. Oitmaa, Phys. Rev. B 60, 7278 (1999)

${ }^{11}$ N. Read and S. Sachdev, Phys. Rev. Lett. 62, 1694 (1989); N. Read and S. Sachdev, Phys. Rev. B 42, 4568 (1990); N. Read and S. Sachdev, Phys. Rev. Lett. 66, 1773 (1991); S. Sachdev and N. Read, Int. J. Mod. Phys. B 5, 219 (1991); S. Sachdev,
Quantum Phase Transitions (Cambridge University Press, Cambridge, England, 1999).

${ }^{12}$ D.M. Ceperley and B.J. Alder, Phys. Rev. Lett. 45, 566 (1980); D.M. Ceperley, Rev. Mod. Phys. 67, 279 (1995).

${ }^{13}$ We are grateful to Steve White for making his software available to us.

${ }^{14}$ M.S.L. du Croo de Jongh, Ph.D. thesis Leiden University, 1999, cond-mat/9908200 (unpublished).

${ }^{15}$ H.J.M. van Bemmel, D.F.B. ten Haaf, W. van Saarloos, J.M.J. van Leeuwen, and G. An, Phys. Rev. Lett. 72, 2442 (1994).

${ }^{16}$ D.F.B. ten Haaf, H.J.M. van Bemmel, J.M.J. van Leeuwen, W. van Saarloos, and D.M. Ceperley, Phys. Rev. B 51, 13039 (1995).

${ }^{17}$ S. Sorella and L. Capriotti, Phys. Rev. B 61, 2599 (2000).

${ }^{18}$ N. Trivedi and D.M. Ceperley, Phys. Rev. B 41, 4552 (1990).

${ }^{19}$ W. Marshall, Proc. R. Soc. London, Ser. A 232, 48 (1955).

${ }^{20}$ The value for $m=150$ is not in line with the others. This can be explained by the fact that the construction of this DMRG wave function was slightly different from the others in which the basis was built up gradually.

${ }^{21}$ S Östlund and S. Rommer, Phys. Rev. Lett. 75, 3537 (1995); S. Rommer and S. Östlund, Phys. Rev. B 55, 2164 (1997). 